UDK 517.54

E. G. Ganenkova, V. V. Starkov

\title{
ASYMPTOTIC VALUES OF FUNCTIONS, ANALYTIC IN PLANAR DOMAINS ${ }^{1}$
}

\begin{abstract}
In [1] W. Gross constructed the example of an entire function of infinite order whose set of asymptotic values is equal to the extended complex plain. We obtain an analog of Gross' result for functions, analytic in planar domains of arbitrary connectivity with isolated boundary fragment.
\end{abstract}

Key words: analytic function, asymptotic value, isolated boundary fragment.

2010 Mathematical Subject Classification: 30D40.

In $1918 \mathrm{~W}$. Gross constructed the example of an entire function $\Phi$ of infinite order whose set of asymptotic values is equal to $\overline{\mathbb{C}}$ (see [1]). This means that for every $a \in \overline{\mathbb{C}}$ there exists an open arc $\Gamma_{a}$ with endpoint at infinity such that

$$
\lim _{\Gamma_{a} \ni z \rightarrow \infty} \Phi(z)=a .
$$

In this note, using Gross' example, we construct a function, analytic in preassigned planar domain, having $\overline{\mathbb{C}}$ as the set of asymptotic values at a given boundary point.

Let $D$ be an arbitrary domain, $D \subset \mathbb{C}, z_{0} \in \partial D$ is an accessible point (i. e. there exists an open arc $\Gamma \subset D$ with endpoint $z_{0}$ ), $f$ is analytic in $D$ function.

Definition 1. [2], [3, p. 8] We say that $a \in \overline{\mathbb{C}}$ is an asymptotic value of $f$ at the point $z_{0}$ if there exists an open arc $\Gamma_{a} \subset D$ with endpoint $z_{0}$ such that

$$
\lim _{\Gamma_{a} \ni z \rightarrow z_{0}} f(z)=a .
$$

\footnotetext{
${ }^{1}$ This work was supported by the Programm of strategic development of the PetrSU and Russian Foundation for Basic Research (project No. 11-01-00952-a).

(C) Ganenkova E. G., Starkov V. V., 2013
} 
We shall say that $\Gamma_{a}$ is an asymptotic curve corresponding to the asymptotic value $a$. The set of all asymptotic values of a function $f$ at a point $z_{0}$ we shall denote by $\operatorname{As}\left(f, z_{0}\right)$.

This definition restricts the choice of point $z_{0}$. It is possible to define asymptotic values of function not at all boundary points. In the sequel, we assume that $z_{0}$ belongs to an isolated boundary fragment.

Definition 2. [4] A domain $D \subset \mathbb{C}$ has an isolated boundary fragment if one of the following conditions holds:

(I) There exists a continuum $K \subset \partial D$, different from a point, and an open set $U$ such that $K \subset U$ and $(\partial D \backslash K) \cap U=\emptyset$.

(II) There exists a Jordan arc $\Gamma \subset \partial D$ with distinct ends $\xi, \eta$ and an open disk $B$ such that $\xi, \eta \in \partial B, \Gamma \backslash\{\xi, \eta\} \subset B$ and $(\partial D \backslash \Gamma) \cap B=\emptyset$.

(III) There exist a point $a \in \partial D$ and an open disc $B(a)$ centered at $a$ such that $(B(a) \backslash\{a\}) \cap \partial D=\emptyset$, i. e. $a$ is an isolated point of the set $\partial D$.

Using Gross' example we construct a functions $f=\Phi \circ \varphi$, analytic in a domain $D$ with isolated boundary fragment, with condition

$$
\operatorname{As}\left(f, z_{0}\right)=\overline{\mathbb{C}}, \quad z_{0} \in \partial D .
$$

Here $\Phi$ is an entire function, $\varphi$ is either injective analytic function from $D$ into $\mathbb{C}$ or surjective at most 3 -valent analytic function from $D$ onto $\mathbb{C}$.

Theorem 1. Let $D$ be an arbitrary domain with isolated boundary fragment and a point $z_{0}$ belong to this fragment. If it is the fragment of type (I) we assume in addition that $z_{0}$ is an accessible point and it corresponds to the prime end, which is equal to $z_{0}$. Then there exist an injective analytic function $\varphi: D \rightarrow \mathbb{C}$ and an entire function $\Phi$ such that

$$
\operatorname{As}\left(\Phi \circ \varphi, z_{0}\right)=\overline{\mathbb{C}} \quad \text { and } \quad \varlimsup_{z \rightarrow z_{0}} \frac{\ln \ln |\Phi(\varphi(z))|}{\ln |\varphi(z)|}=\infty .
$$

Proof. Let $\Phi$ be Gross' function. Asymptotic curves $\Gamma_{a}$, corresponding to asymptotic values $a \in \overline{\mathbb{C}}$ of function $\Phi$, are rays, lying in the set

$$
\{z \in \mathbb{C}:|\arg z| \leq \pi / 4 \text { or }|\pi-\arg z| \leq \pi / 4\}
$$

(see [1]).

Case 1. If $z_{0}$ is an isolated boundary fragment of type (III) we take

$$
\varphi(z)=\frac{1}{z-z_{0}}
$$


By $\gamma_{a}$ denote a connected subset of $\varphi^{-1}\left(\Gamma_{a} \cap \varphi(D)\right)$ with endpoint at $z_{0}$. Then

$$
\lim _{\gamma_{a} \ni z \rightarrow z_{0}} \Phi(\varphi(z))=\lim _{\Gamma_{a} \ni w \rightarrow \infty} \Phi(w)=a \quad \forall a \in \overline{\mathbb{C}}
$$

and

$$
\varlimsup_{\gamma_{a} \ni z \rightarrow z_{0}} \frac{\ln \ln |\Phi(\varphi(z))|}{\ln |\varphi(z)|}=\varlimsup_{w \rightarrow \infty} \frac{\ln \ln |\Phi(w)|}{\ln |w|}=\infty .
$$

Case 2. Let $z_{0}$ belong to an isolated boundary fragment $\Gamma$ of type (II). $\Gamma$ can be considered as an open arc. By the Riemann mapping theorem there exists a biholomorphic mapping $\psi$ from $D$ into the upper half-plane $P$ such that

$$
\{z \in \mathbb{C}:|z|<\rho, \operatorname{Im} z>0\} \subset D_{1}=\psi(D) \text { for some } \rho>0,
$$

the point $0 \in \partial D_{1}$ corresponds to the $z_{0}$ and some interval $(\alpha, \beta) \ni 0$ corresponds to $\Gamma$ (see [4] for details). It follows from the Caratheodory theorem that $\psi$ can be extended to homeomorphism from $(D \cup \Gamma)$ onto $(\psi(D) \cup(\alpha, \beta))$.

The univalent in $D_{1}$ function

$$
w_{ \pm}=\frac{ \pm i}{z^{2}}
$$

(the sign will be chosen later) maps $D_{1}$ onto a domain $D_{2}$, containing the rays

$$
\Gamma_{a}^{\prime}=\Gamma_{a} \cap\left\{w \in \mathbb{C}:|w| \geq \frac{1}{\rho^{2}}\right\} \quad \forall a \in \overline{\mathbb{C}} .
$$

Since $\Phi$ is the function of infinite order then there exists a sequence $w_{n} \rightarrow \infty$ as $n \rightarrow \infty$ such that

$$
\lim _{n \rightarrow \infty} \frac{\ln \ln \left|\Phi\left(w_{n}\right)\right|}{\ln \left|w_{n}\right|}=\infty .
$$

We can choose a subsequence $w_{n}$ (let us save the notation) that doesn't belong to one of the following sets $\{w \in \mathbb{C}: \operatorname{Re} w=0, \operatorname{Im} z \geq 0\}$ or $\{w \in \mathbb{C}: \operatorname{Re} w=0, \operatorname{Im} z \leq 0\}$. Therefore, we can choose the sign for the function $w_{ \pm}$such that $w_{n} \in D_{2}$.

Let $\varphi$ be the constructed above univalent function from $D$ onto $D_{2}$, $\gamma_{a}=\varphi^{-1}\left(\Gamma_{a}^{\prime}\right), z_{n}=\varphi^{-1}\left(w_{n}\right)$. Then $\operatorname{As}\left(\Phi \circ \varphi, z_{0}\right)=\overline{\mathbb{C}}$ and

$$
\lim _{n \rightarrow \infty} \frac{\ln \ln \left|\Phi\left(\varphi\left(z_{n}\right)\right)\right|}{\ln \left|\varphi\left(z_{n}\right)\right|}=\infty .
$$


Case 3. Let $z_{0}$ belong to isolated boundary fragment $K$ of type (I). Consider the simply connected domain $D_{0} \supset D, \partial D_{0}=K$. By the Riemann mapping theorem there exists a univalent conformal mapping $\psi$ of the domain $D_{0}$ onto the upper half-plane $P$. In addition, the origin corresponds to the accessible boundary point $z_{0} \in \partial D_{0}$. It follows from the definition of isolated boundary fragment of type (I) that if $D_{1}=\psi(D)$, then the set $\partial D_{1} \backslash \mathbb{R}$ is a subset of some compact set $C \subset P$. Hence, we can find $\rho>0$ such that

$$
D_{1} \supset\{z \in \mathbb{C}:|z|<\rho, \operatorname{Im} z>0\} .
$$

Since the point $z_{0}$ is a prime end of the domain $D$ (this means that $z_{0}$ is a prime end of $D_{0}$ ), then $\psi^{-1}$ can be continuously extended from $D_{1}$ to $D_{1} \cup\{0\}, \psi^{-1}(0)=z_{0}$ (see [5, ch. II, $\S 3$, p. 41]). Consequently, $\psi$ is a homeomorphism from $D \cup\left\{z_{0}\right\}$ to $D_{1} \cup\{0\}$. Now, as in Case 2, we put $\varphi=w_{ \pm} \circ \psi$, choosing an appropriate sign. Then for all $a \in \overline{\mathbb{C}}$ conditions (1) and (2) are fulfilled for $\varphi$ and $\gamma_{a}=\varphi^{-1}\left(\Gamma_{a}^{\prime}\right)$.

It was shown in [4] that the domain $D$ from Theorem 1 can be mapped onto $\mathbb{C}$ locally biholomorphically and at most 3 -valently. The mapping function $F: D \rightarrow \mathbb{C}$ is the composition of the univalent function $\varphi$ from Theorem 1 and an 3-valent function $g$. Here 3 -valence of a function $F$ means that for every $w \in \mathbb{C}$ the equation $F(z)=w$ has at most three solutions and it has exactly three solutions for some $w \in \mathbb{C}$. For the polynomial $Q(z)=z^{3}-3 z$ the Riemann surface $Q(\mathbb{C})$ contains all rays $\Gamma_{a}^{\prime}=\Gamma_{a} \cap\{w \in \mathbb{C}:|w|>\rho\}$ for sufficiently large $\rho>2$, here $\Gamma_{a}$ are rays from Gross' example. Thus, it follows from the construction of mapping $g$ that the rays $\Gamma_{a}^{\prime}$ belong to the Riemann surface $F(D)$. Like in the proof of Case 3, Theorem 1 we can show that $F$ is continuous at the prime end $z_{0}$ and

$$
\lim _{D \ni z \rightarrow z_{0}} F(z)=\infty .
$$

Therefore, for every $a \in \overline{\mathbb{C}}$ there exists an open arc $\gamma_{a} \subset D, F\left(\gamma_{a}\right)=\Gamma_{a}^{\prime}$ such that

$$
\lim _{\gamma_{a} \ni z \rightarrow z_{0}} \Phi(F(z))=\lim _{\Gamma_{a} \ni w \rightarrow \infty} \Phi(w)=a
$$

and

$$
\varlimsup_{z \rightarrow z_{0}} \frac{\ln \ln |\Phi(F(z))|}{\ln |F(z)|}=\infty
$$


Consequently, the univalent function from Theorem 1 can be replaced by the at most 3 -valent function $F$ (it was proved in [6] that there is no a 2-valent locally biholomorphic mapping of $D$ onto $\mathbb{C}$ ).

In such way we have obtained

Theorem 2. Let $D$ and $z_{0}$ be as in Theorem $1, D \neq \mathbb{C} \backslash\left\{z_{0}\right\}$. Then there exist a locally biholomorphic at most 3-valent surjective function $F: D \rightarrow \mathbb{C}$ and an entire function $\Phi$ of infinite order such that

$$
\operatorname{As}\left(\Phi \circ F, z_{0}\right)=\overline{\mathbb{C}} \quad \text { and } \quad \varlimsup_{z \rightarrow z_{0}} \frac{\ln \ln |\Phi(F(z))|}{\ln |F(z)|}=\infty
$$

\section{References}

[1] Gross W. Eine ganze Funktion für die jede Komplexe Zahl Konvergenzwert ist // Math. Ann. 1918. V. 79. P. 201-208.

[2] Encyclopedia of Mathematics. [S. 1.]: Kluwer Academic Publishers, 1987. Vol. 1 (A-B).

[3] Collingwood E. F., Lohwater A. J. The theory of cluster sets. Cambridge: Collingwood Lohwater Cambridge University Press, 1966.

[4] Liczberski P., Starkov V. V. On locally biholomorhic mappings from multi-connected onto simply connected domains // Ann. Polon. Math. 2005. V. 85. No. 2. P. 135-143.

[5] Goluzin G. M. Geometric Theory of Functions of a Complex Variable. Providence, R. I.: American Mathematical Scociety, 1969.

[6] Starkov V. V. Locally biholomorphic mappings of multiconnected domains // Sib. Math. J. 2007. V. 48. No. 4. P. 733-739.

The work is received on May 25, 2013.

Petrozavodsk State University,

Department of Mathematics

185910, Petrozavodsk, Lenin Avenue, 33.

E-mail: g_ek@inbox.ru, vstar@psu.karelia.ru 